\title{
Macroalgas de riachos na Serra do Japi, sudeste do Estado de São Paulo, Brasil
}

\author{
(10)Aurelio Fajar Tonetto ${ }^{1,3}$, Marina Ramos Auricchio ${ }^{2}$, Luigi Cezare Pezzatto ${ }^{1}$ e Cleto Kaveski Peres ${ }^{2}$
}

Recebido: 26.03.2018; aceito: 8.08.2018

\begin{abstract}
Stream macroalgae from Serra do Japi, Southeast of São Paulo State, Brazil). Diversity of lotic macroalgae has been scarcely studied in Brazil. Floristic studies have not been recently published with a lack of information about ecology and taxonomy of these groups. Therefore, the present work aimed to survey macroalgae species from streams of the Serra do Japi, an area of Semideciduous Seasonal Forest ( $23^{\circ} 12^{\prime}-23^{\circ} 21^{\prime} \mathrm{S}$ and $\left.46^{\circ} 30^{\prime}-46^{\circ} 05^{\prime} \mathrm{W}\right)$. We sampled 16 streams and we identified 16 taxa (11 infrageneric, 4 generic and one life stage, 'Chantransia'), distributed in four divisions. Cyanobacteria was the best well represented group, with about $38 \%$ of the species, followed by Rhodophyta with approximately $31 \%$ of the taxa sampled. The sporophyte stage 'Chantransia' and Phormidium aerugineocaeruleum were the most widespread, occurring in $50 \%$ and $29 \%$ of the streams investigated, respectively. The knowledge about the composition and occurrence of macroalgae species can provide valuable information for decision-making on conservation of water resources, particularly in protected areas as Serra do Japi.
\end{abstract}

Keywords: conservation, lotic algae, atlantic rainforest, taxonomy

RESUMO - (Macroalgas de riachos na Serra do Japi, sudeste do Estado de São Paulo, Brasil). A diversidade de macroalgas lóticas tem sido pouco estudada no Brasil. Levantamentos florísticos não têm sido publicados, gerando um déficit de informações sobre a ecologia e taxonomia desses grupos. Sendo assim, o presente trabalho teve como objetivo realizar o levantamento de espécies de macroalgas de riachos da Serra do Japi, uma área de Floresta Estacional Semidecidual $\left(23^{\circ} 12^{\prime}\right.$ $23^{\circ} 21^{\prime} \mathrm{S}$ e $46^{\circ} 30^{\prime}-46^{\circ} 05^{\prime} \mathrm{W}$ ). Foram amostrados 16 riachos e foram identificados 16 táxons ( 11 infragenéricos, 4 genéricos e um estágio de vida, 'Chantransia'), distribuídos em quatro divisões. Cyanobacteria foi o grupo melhor representado, com cerca de $38 \%$ das espécies, seguido de Rhodophyta com aproximadamente $31 \%$ dos táxons amostrados. O estágio esporofítico 'Chantransia' e Phormidium aerugineocaeruleum foram os táxons os mais bem distribuídos, ocorrendo em 50\% e $29 \%$ dos pontos investigados, respectivamente. O conhecimento sobre a composição e ocorrência das espécies de macroalgas pode trazer informações valiosas para a tomada de decisões sobre a conservação de recursos hídricos, particularmente em áreas protegidas como a Serra do Japi.

Palavras-chave: conservação, algas lóticas, mata atlântica, taxonomia, conservação

\section{Introdução}

As algas bentônicas de riachos exercem um papel fundamental como produtoras primárias nesses ambientes (Brito et al. 2006, Branco et al. 2017). Conhecer a diversidade desses organismos e os seus aspectos ecológicos é essencial para determinar o manejo e conservação das espécies, contribuindo assim para o gerenciamento dos recursos hídricos (Dubois 2003). Nesse contexto, diversos estudos foram realizados, especialmente com espécies de macroalgas lóticas, a fim de conhecer de forma mais precisa a composição dessas comunidades, no Brasil e no mundo (e.g. Sheath et al. 1989, Sheath \& Cole 1992, Branco \& Necchi 1996, 1998, Necchi et al. 2000, Sherwood \& Sheath 1999, Hu \& Xie 2006).

Entretanto, a maioria dos esforços esteve concentrada na década de 1990 (Dias 1992, Necchi et al. 1995, Branco \& Necchi 1996, Branco et al. 1999 , Sherwood \& Sheath 1999) e 2000 (Necchi et al. 2000, Branco et al. 2005, Krupek et al. 2008, Peres et al. 2008). Sendo assim, nos últimos anos, principalmente no Brasil, trabalhos envolvendo levantamentos taxonômicos de espécies de macroalgas lóticas têm

1. Universidade Paulista, Departamento de Biologia, Av. Armando Giassetti, 577, Vila Hortolândia, 13214-525, Jundiaí, São Paulo, Brasil

2. Universidade Federal da Integração Latino Americana, Instituto Latino Americano da Vida e da Natureza, Av. Tancredo Neves, 6731, Parque Tecnológico Itaipu, 85867970, Foz do Iguaçu, Paraná, Brasil

3. Autor para correspondência: tonettoaf@gmail.com 
sido ainda escassos. Os trabalhos mais recentes têm envolvido principalmente abordagens experimentais (Branco et al. 2010, 2017, Tonetto et al. 2014, 2015) ou ecológicas (Oliveira et al. 2013, Branco et al. 2017, Peres et al. 2017).

Infelizmente, a diversidade biológica encontra-se ameaçada devido às ações antrópicas (Brown \& Lomolino 1998). A perda e degradação de habitat, a introdução de espécies exóticas e poluição ambiental são exemplos de ações que aceleram o processo de extinção das espécies (Wilson 1992, Hooper et al. 2005, Sodhi \& Ehrlich 2010). Nesse contexto, o grande problema para a biodiversidade encontra-se nas quantidades alarmantes de espécies que desaparecem antes mesmo de serem descobertas e descritas (Dubois 2003, Loreau et al. 2006). Assim, as dificuldades enfrentadas na construção de estratégias eficientes para a conservação podem estar relacionadas ao nosso conhecimento ainda limitado sobre a biodiversidade, além do evidente impacto crescente dos efeitos antrópicos (Dubois 2003).

De acordo com esse cenário, uma estratégia para a conservação das espécies é aumentar efetivamente nossas informações sobre a taxonomia e distribuição espacial das mesmas. Dado que muitas espécies ainda não foram descobertas (Déficit Linneano, Bini et al. 2006) e que mesmo as espécies já descritas ainda possuem distribuição espacial pouco conhecida (Déficit Wallaceano, Bini et al. 2006), trabalhos envolvendo o levantamento taxonômico de regiões ainda não investigadas tornam-se fundamentais para reduzir esses déficits em biodiversidade (Mace 2004).

A Serra do Japi, por exemplo, caracteriza-se por ser um raro remanescente de mata atlântica que apresenta rica biodiversidade, uma vez que se encontra entre duas diferentes formações florestais que proporcionam uma grande riqueza de formas de vida (Cardoso-Leite et al. 2002). Nesse contexto, a região apresenta potencial para se reduzir o déficit de conhecimento em biodiversidade de macroalgas lóticas, uma vez que apresenta rica disponibilidade de águas dentro da reserva e entornos (CardosoLeite 2000). Entretanto, não há trabalhos envolvendo a caracterização taxonômica das espécies desses organismos na reserva do Japi.

Considerando a atual carência de estudos com macroalgas lóticas no Brasil e na região, o presente estudo tem como objetivo conhecer a biodiversidade das comunidades de macroalgas dos ambientes lóticos de uma área de Floresta Estacional Semidecidual. Assim, o trabalho propõe a realização de um levantamento taxonômico de macroalgas dos riachos da Serra do Japi e entornos (redução do Deficit Linneano), amostrando e caracterizando os ambientes ocupados pelas espécies de uma região nunca investigada até o presente momento (redução do Deficit Wallaceano). Por fim, os dados levantados com esse trabalho podem contribuir com a produção de informações valiosas para as estratégias de conservação de algas de ambientes lóticos.

\section{Material e métodos}

Área de estudo - A Serra do Japi (Parque Estadual da Serra do Japi) localiza-se entre quatro municípios pertencentes ao Estado de São Paulo: Jundiaí, Pirapora do Bom Jesus, Cajamar e Cabreúva (figura 1). A Serra está posicionada entre $23^{\circ} 12^{\prime}-23^{\circ} 21^{\prime} \mathrm{S}$ e $46^{\circ} 30^{\prime}$ $46^{\circ} 05^{\prime} \mathrm{W}$ e apresenta aproximadamente 35.000 ha. de extensão coberta por Floresta Estacional Semidecidual, a qual representa a principal fitofisionomia presente na região (Cardoso-Leite et al. 2002).

A região está localizada na Província geomorfológica do Planalto Atlântico, na Zona de Serrania

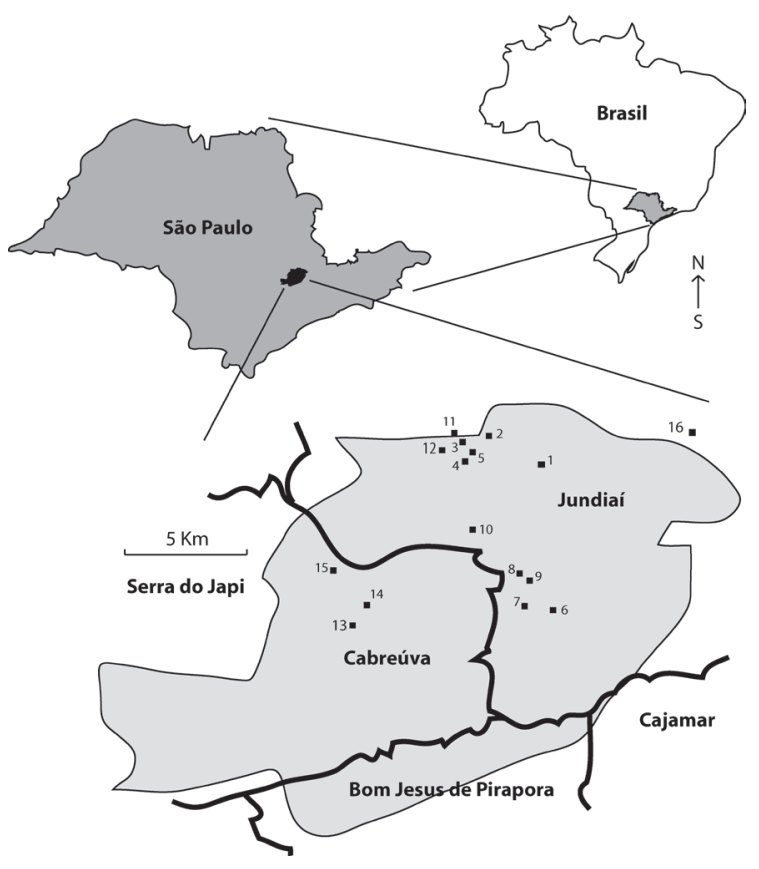

Figura 1. Localização geral da Serra do Japi, entre os municípios de Jundiaí, Cabreúva, Bom Jesus de Pirapora e Cajamar, Estado de São Paulo, Sudeste do Brasil. Em detalhe a localização dos 16 pontos de amostragem utilizados no estudo sobre as espécies de macroalgas. Barra de escala $=5 \mathrm{Km}$.

Figure 1. Location of Serra do Japi, inserted in Jundiaí, Cabreúva, Bom Jesus de Pirapora e Cajamar, State of São Paulo, Brasil. southeast of Brazil. In detail there is the location of the 16 sampling points used in this study about macroalgal species. Scale bar = $5 \mathrm{Km}$. 
de São Roque (Muccioli 1993, Saka 2009). O relevo apresenta características montanhosas, com declividades desde médias até altas (Saka 2009). A altitude média do município é de aproximadamente $762 \mathrm{~m}$., mas com picos mais altos de até 1200/1300 m nas áreas de Serras (Cardoso-Leite, 2000). Com declividades superiores a $20 \%$ e em grande parte maiores até que $40 \%$, esta área apresenta grande potencial erosivo (Muccioli 1993).

Os tipos de clima predominantes na Serra do Japi são Cfa e Cfb (sistema internacional de Köeppen), ou seja, climas quentes e úmidos, sem estação seca, com chuvas acima de $30 \mathrm{~mm}$ nos períodos mais secos (Cardoso-Leite et al. 2000, Saka 2009). Segundo Pinto (1992) a precipitação média varia de $41 \mathrm{~mm} / \mathrm{mês}$ nos meses secos e até $250 \mathrm{~mm} / \mathrm{mês}$ nos chuvosos.

Por fim, a região apresenta grande riqueza de mananciais pertencentes à bacia dos rios Piracicaba, Capivari e Jundiaí. O nível de preservação é alto dentro da reserva, sendo presente inúmeros riachos de águas cristalinas, de alta qualidade para abastecimento do município de Jundiaí.

Procedimentos de amostragem - As amostragens foram realizadas através da técnica da transeção (consistindo de segmentos de 10 metros de comprimento em cada ponto amostral) (de acordo com Necchi et al. 1995) em 16 riachos de $1^{\mathrm{a}}$ a $3^{\mathrm{a}}$ ordem (classificação de Strahler 1957), localizados dentro (e entornos) do Parque Estadual da Serra do Japi, Jundiaí, São Paulo, Brasil (figura 1). Cada ponto de amostragem foi visitado uma vez, nas épocas sazonais de menor nível de precipitação pluviométrica (i.e. final do outono ao início da primavera), período descrito como sendo o de maior diversidade e abundância de espécies de macroalgas lóticas nas regiões Sul e Sudeste do país (Necchi et al. 2000, Branco et al. 2009). Um conjunto de parâmetros ambientais foi amostrado nos riachos no momento da coleta das macroalgas. Profundidade e largura médias foram estimados em campo com fita métrica, enquanto a velocidade da correnteza foi mensurada pelo método do objeto flutuante. Turbidez, $\mathrm{pH}$, cor, condutividade específica, nitrogênio total ( $\mathrm{N}$ total), fósforo total (P total) e fosfato foram mensurados, a partir de uma amostra coletada no ponto médio do riacho, no laboratório do Departamento de Água e Esgoto de Jundiaí, de acordo com os métodos da APHA (1998).
Neste estudo adotou-se a definição de Sheath \& Cole (1992), na qual macroalgas são consideradas aquelas com estrutura do talo visível a olho nu. Os espécimes de macroalgas coletadas nos pontos de amostragem foram preservados em solução de formaldeído tamponado 4\%. Amostras-testemunho de cada população encontrada foram incorporadas ao herbário da Universidade Estadual do Oeste do Paraná (UNOP), em meio líquido. As observações microscópicas e as análises morfométricas foram realizadas no laboratório da UNILA (Foz do Iguaçu), com auxílio de microscópio trinocular Zeiss, Axiolab A1 portando um sistema de captura e processamento de imagem.

A classificação das espécies em divisões seguiu o sistema de Graham et al. (2009), para classes e níveis inferiores foi utilizado o sistema de Guiry \& Guiry (2018).

Para todos os táxons amostrados são apresentados uma breve descrição e fotomicrografias. As medidas de caracteres morfométricos $(n>10)$ como diâmetro (diâm.) e comprimento (compr.) das células (ou estruturas) são apresentadas para todos os táxons, ao passo que a relação comprimento/diâmetro celular (compr./diâm.) foi apresentada, sempre que necessário. Comentários taxonômicos, quando necessários, incluem aspectos da caracterização e/ou dificuldades na identificação. São apresentados, ainda, os pontos de amostragem onde ocorreram na Serra do Japi e as características físicas e químicas dos riachos onde ocorreram.

\section{Resultados}

Foram identificados 16 táxons (11 infragenéricos, 4 genéricos e uma fase do ciclo de vida de Batrachospermum spp., 'Chantransia', distribuídos em quatro divisões. Cyanobacteria foi o grupo melhor representado, com cerca de $38 \%$ das espécies, seguido de Rhodophyta com aproximadamente $31 \%$ dos táxons amostrados, Chlorophyta com $25 \%$ e Ochrophyta com $6 \%$. O estágio esporofítico de Batrachospermales 'Chantransia' e Phormidium aerugineocaeruleum foram os mais amplamente distribuídos, ocorrendo em $50 \%$ e $29 \%$ dos pontos investigados, respectivamente.

Chave artificial para identificação dos táxons de macroalgas na Serra do Japi:

1. Cianobactérias, conteúdo celular homogêneo, sem cloroplastos

2. Filamentos não ramificados, homocitadas

3. Presença de mais de um tricoma em uma mesma bainha, célula apical cônica: Microcoleus subtorulosus

3. Tricoma único por bainha, célula apical truncada ou arredondada 
4. Células com diâmetro inferior a $3 \mu \mathrm{m}$ : Phormidium sp.

4. Células com diâmetro superior a $3 \mu \mathrm{m}$

5. Septos celulares distintos, conteúdo celular azul opaco com grânulos escuros e pequenos: Phormidium retzii

5. Septos celulares indistintos, conteúdo celular azul brilhante com grânulos esverdeados pequenos e grandes: Phormidium aerugineocaeruleum

2. Filamentos ramificados, heterocitadas

6. Ramificações falsas, unisseriado: Scytonema arcangeli

6. Ramificações verdadeiras, multisseriado: Stigonema mamillosum

1. Algas eucarióticas, presença de cloroplastos

7. Filamentos falsos, formados por arranjo colonial, quebradiços: Hydrosera whampoensis

7. Filamentos verdadeiros

8. Filamentos curtos e prostrados formando crostas: Hildenbrandia angolensis

8. Filamentos longos e eretos com a maior porção livre na coluna d'água

9. Filamentos não ramificados

10. Cloroplastos centrais arredondados, dois por célula, arredondados e com um pirenóide cada: Zygogonium sp.

10. Cloroplastos parietais laminar ou reticulado com múltiplos pirenóides

11. Células com anéis nos septos: Oedogonium sp.

11. Células sem anéis nos septos: Klebsormidium fluitans

9. Filamentos ramificados

12. Filamentos multisseriados corticados

13. Não verticilados: Compsopogon caeruleus

13. Verticilados

14. Ramos dos verticilos com no máximo 7 células: Batrachospermum sp. 1

14. Ramos dos verticilos com mais de 8 células: Batrachospermum sp. 2

12. Filamentos unisseriados ecorticados

15. Cloroplastos verdes, sem monosporângios: Stigeoclonium amoenum

15. Cloroplastos arroxeados, com monosporângios: Estágio 'Chantransia'

Descrição dos táxons encontrados na Serra do Japi, São Paulo, sudeste do Brasil:

Chlorophyta, Chlorophyceae, Chaetophoraceae, Stigeoclonium

Stigeoclonium amoenum Kützing, Phyc. Gen., 198, 1845.

Figuras 2, 3

Filamentos ramificados formando tufos verdes brilhantes; sistema prostrado formado por uma massa de rizoides, sistema ereto bem desenvolvido; células

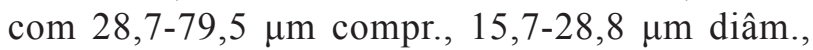
compr./diâm. 1,6-2,9; presença de ramificações alternadas e opostas; células dos ramos em geral mais curtas e doliformes; ápices dos ramos principais acuminados; cloroplastos parietais em forma de banda mediana.

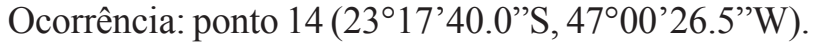
BRASIL. São Paulo: Cabreúva, Serra do Japi, 14-V-2016, A.F. Tonetto s.n. (UNOP5344).
Características do riacho $(\mathrm{n}=1)$ : turbidez $0,8 \mu \mathrm{T}$; cor 19 $\mu \mathrm{H} ; \mathrm{pH} 6,8$; condutividade $21 \mu \mathrm{S}$; N Total $0,0 \mathrm{mg} / \mathrm{L} ; \mathrm{P}$ Total $0,04 \mathrm{mg} / \mathrm{L}$; fosfato $0,011 \mathrm{mg} / \mathrm{LPO}_{4}$; profundidade $29,4 \mathrm{~cm}$, largura $307,2 \mathrm{~cm}$; velocidade da correnteza $0,51 \mathrm{~m} / \mathrm{s}$ e ambiente parcialmente sombreado.

Oedogoniaceae, Oedogonium

\section{Oedogonium sp.}

Figura 4

Filamentos unisseriados simples, aderidos a rochas; presença de anéis nos septos formados por quebra durante as divisões celulares; células cilíndricas, 50,6-78,3 $\mu \mathrm{m}$ compr., 13,9-17,5 $\mu \mathrm{m}$ diâm., compr./diâm. 3,2-4,7; cloroplastos reticulados com muitos pirenoides; oogônios e anterídios não observados.

Comentários taxonômicos: O material analisado constitui-se unicamente de filamentos estéreis. Como a identificação específica está baseada nas características 

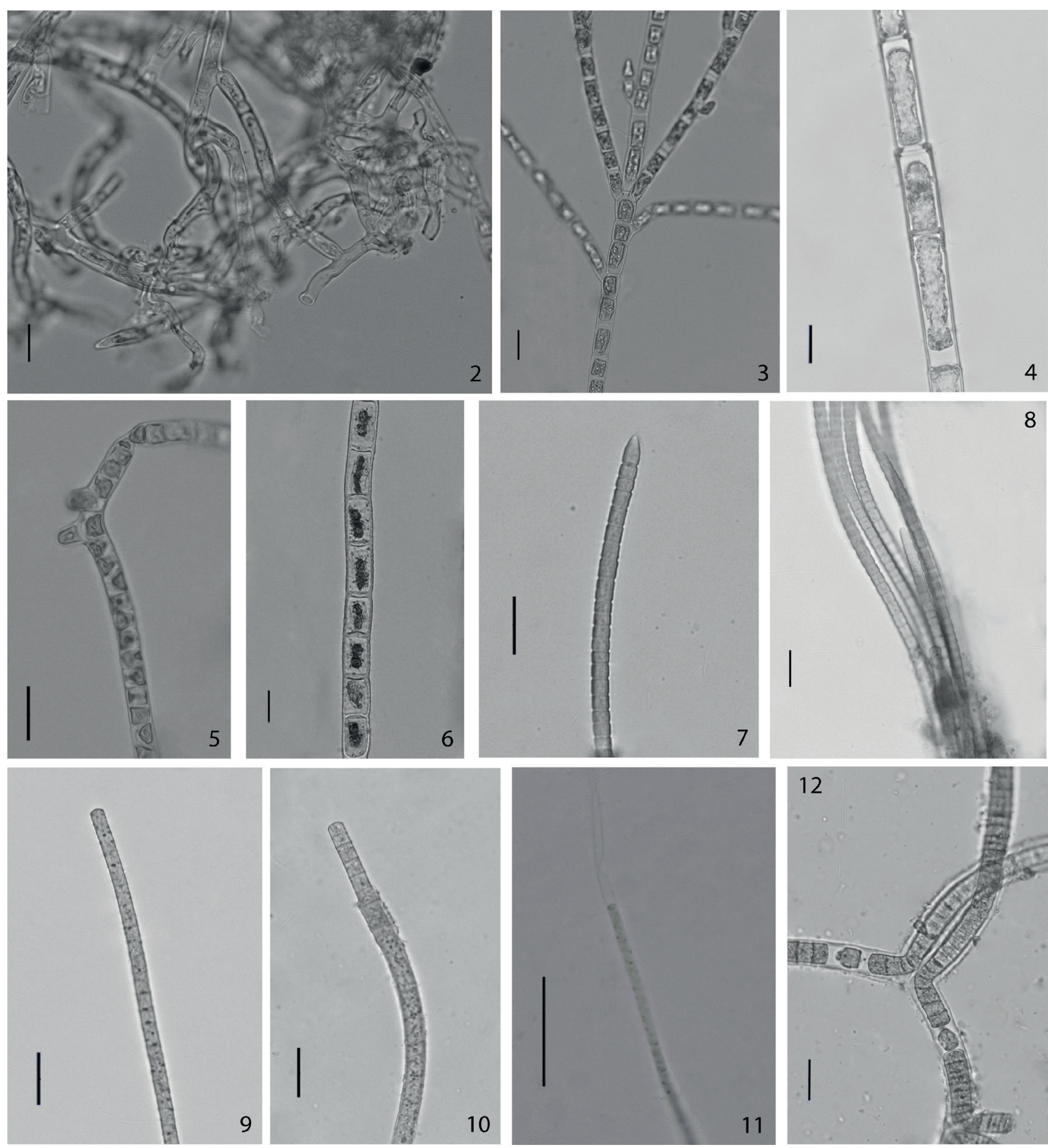

Figuras 2-12. Espécies de Chlorophyta e Cianobacteria da Serra do Japi. 2-3. Stigeoclonium amoenum. 2. Sistema basal. 3. Sistema ereto. 4. Oedogonium sp.. 5. Klebsormidium fluitans. 6. Zygogonium sp. 7-8. Microcoleus subtorulosus. 7. Tricoma com célula apical cônica. 8. Filamento com vários tricomas. 9. Phormidium aerugineocaeruleum, septos indistintos. 10. Phormidium retzii, septos distintos. 11. Phormidium sp. 12. Scytonema arcangeli, ramificação falsa. Barra de escala $=20 \mu \mathrm{m}$.

Figures 2-12. Figures 2-12. Species of Chlorophyta and Cyanobacteria from Serra do Japi. 2-3. Stigeoclonium amoenum. 2. Basal system. 3. Erect system. 4. Oedogonium sp.. 5. Klebsormidium fluitans. 6. Zygogonium sp. 7-8. Microcoleus subtorulosus. 7. Trichome with conical apical cell. 8. Filament with several trichomes. 9. Phormidium aerugineocaeruleum, indistinct septum. 10. Phormidium retzii, distinct septum. 11. Phormidium sp. 12. Scytonema arcangeli, false branching. Scale bar $=20 \mu \mathrm{m}$. 
reprodutivas só foi possível a identificação em nível genérico.

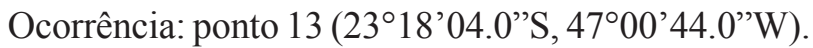
BRASIL. São Paulo: Cabreúva, Serra do Japi, 14-V-2016, A.F. Tonetto s.n. (UNOP5341).

Características do riacho $(\mathrm{n}=1)$ : turbidez $0,7 \mu \mathrm{T}$; cor $22 \mu \mathrm{H}$; pH 6,7; condutividade $15 \mu \mathrm{S}$; N Total $0,0 \mathrm{mg} / \mathrm{L}$; P Total $0,1 \mathrm{mg} / \mathrm{L}$; fosfato $0,016 \mathrm{mg} / \mathrm{L} \mathrm{PO}_{4}$; profundidade $7,6 \mathrm{~cm}$, largura $103,8 \mathrm{~cm}$; velocidade da correnteza $0,77 \mathrm{~m} / \mathrm{s}$ e ambiente sombreado.

Klebsormidiophyceae, Klebsormidiaceae, Klebsormidium.

Klebsormidium fluitans (F.Gay) Lokhorst, Cryptog.

Stud. 5: 20, 1996 EStichococcus fluitans F.Gay. Figura 5

Filamentos emaranhados formando tufos; filamentos unisseriados não ramificados possuindo articulações intercalares em forma de joelho; células cilíndricas levemente constritas ou doliformes, com 7,2-12,4 $\mu \mathrm{m}$ de compr., 6,5-9,2 $\mu \mathrm{m}$ de diâm., compr./ diâm. 0,7-1,6; cloroplasto único por célula e parietal, laminar com pirenoide simples, ocupando quase toda a circunferência da célula; zoosporângios com poro mediano de liberação de zoósporos.

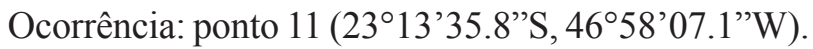
BRASIL. São Paulo: Jundiaí, Serra do Japi, 7-V-2016, A.F. Tonetto s.n. (UNOP5339).

Características do riacho $(\mathrm{n}=1)$ : turbidez $0,6 \mu \mathrm{T}$; cor 9 $\mu \mathrm{H} ; \mathrm{pH} 6,4$; condutividade $17 \mu \mathrm{S} ; \mathrm{N}$ Total $0,0 \mathrm{mg} / \mathrm{L}$; P Total $0,0 \mathrm{mg} / \mathrm{L}$; fosfato $0,032 \mathrm{mg} / \mathrm{L} \mathrm{PO}_{4}$; profundidade $10,2 \mathrm{~cm}$, largura $524 \mathrm{~cm}$; velocidade da correnteza $0,53 \mathrm{~m} / \mathrm{s}$ e ambiente parcialmente sombreado.

Zygnematophyceae, Zygnemataceae, Zygogonium

\section{Zygogonium sp.}

Figura 6

Filamentos unisseriados simples formando emaranhados; células cilíndricas, 15,6-33,6 $\mu \mathrm{m}$ compr., 15,8-19,0 $\mu \mathrm{m}$ diâm., compr./diâm. 0,9-2,0; cloroplastos arredondados, dois por célula, cada um com um pirenoide; zigósporos não observados.

Comentários taxonômicos: o material continha unicamente filamentos estéreis. Como a identificação específica está baseada nas características reprodutivas só foi possível a identificação em nível genérico.
Ocorrência: ponto 10 (2315'50.6”S, 4657'44.6”W). BRASIL. São Paulo: Jundiaí, Serra do Japi, 7-V-2016, A.F. Tonetto s.n. (UNOP5335).

Características do riacho $(\mathrm{n}=1)$ : turbidez $0,5 \mu \mathrm{T}$; cor $46 \mu \mathrm{H}$; pH 4,6; condutividade $27 \mu \mathrm{S}$; N Total $0,8 \mathrm{mg} / \mathrm{L}$; P Total $0,0 \mathrm{mg} / \mathrm{L}$; fosfato $0,042 \mathrm{mg} / \mathrm{L} \mathrm{PO}_{4}$; profundidade $11,4 \mathrm{~cm}$, largura $156 \mathrm{~cm}$; velocidade da correnteza $0,22 \mathrm{~m} / \mathrm{s}$ e ambiente sombreado.

Cyanobacteria, Cyanophyceae, Microcoleaceae, Microcoleus

Microcoleus subtorulosus Gomont ex Gomont, Ann.

Sci. Nat. Bot. Sér. 7, 15: 360, 1892.

Figuras 7, 8

Filamentos emaranhados azul-esverdeados escuro, aderido a substrato rochoso, com 10,8-46,9 $\mu \mathrm{m}$ diâm.; bainha espessa com coloração hialina; tricomas não ramificados paralelos ou levemente torcidos, 1-7 por bainha, constritos nos septos; células azulesverdeadas a roxas, granuladas, 3,8-7,9 $\mu \mathrm{m}$ compr., 5,4-7,1 $\mu \mathrm{m}$ diâm., compr./diâm. 0,6-1,4; célula apical cônico-arredondada ou cônico-aguda, 4,2-9,2 $\mu \mathrm{m}$ compr., 4,5-6,3 ㅆm diâm., compr./diâm. 0,7-1,6.

Ocorrência: ponto 2 (2313'38.4”S, 4657'19.9'W). BRASIL. São Paulo: Jundiaí, Serra do Japi, 9-IV-2016, A.F. Tonetto s.n. (UNOP5321).

Características do riacho $(\mathrm{n}=1)$ : turbidez $1,00 \mu \mathrm{T}$; cor $20 \mu \mathrm{H}$; pH 6,8; condutividade $20,0 \mu \mathrm{S}$; N Total $0,6 \mathrm{mg} / \mathrm{L}$; P Total 0,02 mg/L; fosfato $0,083 \mathrm{mg} / \mathrm{LPO}_{4}$; profundidade $8,2 \mathrm{~cm}$, largura $244,0 \mathrm{~cm}$; velocidade da correnteza $0,30 \mathrm{~m} / \mathrm{s}$ e ambiente sombreado.

Phormidiaceae, Phormidium

Phormidium aerugineocaeruleum (Gomont) Anagnostidis et Komárek, Algolog. Stud. 50-53:407, 1988. $\equiv$ Lyngbya aerugineocaerulea Gomont Figura 9

Filamentos emaranhados azul-esverdeados a azul escuro, com 4,1-5,6 $\mu \mathrm{m}$ diâm.; bainha fina e hialina, homogênea, em geral ausente; tricomas não ramificados, não constritos nos septos; células com septos indistintos, conteúdo azul brilhante com grânulos azul esverdeados pequenos e grandes,

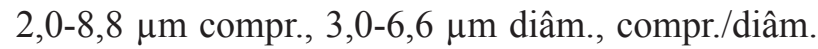
0,4-1,6; célula apical truncada ou arredondada, 2,5-7,1 $\mu \mathrm{m}$ compr., 2,8-5,4 diâm., compr./diâm. $0,8-1,6$. 
Ocorrência: ponto 3 (231'45.1'S, 46 $\left.{ }^{\circ} 57^{\prime} 58.7^{\prime \prime} \mathrm{W}\right)$. BRASil. São Paulo: Jundiaí, Serra do Japi, 9-IV-2016, A.F. Tonetto s.n. (UNOP5324); ponto $6\left(23^{\circ} 17^{\prime} 39.0^{\prime} \mathrm{S}, 46^{\circ} 55^{\prime} 40.5^{\prime} \mathrm{W}\right)$, ponto 7 (2317'22.4”S, 46 56'25.9” W), ponto 11 (2313'35.8”S, 4658'07.1”'W). BRASIL. SÃo PAULO: Jundiaí, Serra do Japi, 7-V-2016, A.F. Tonetto s.n. (UNOP5329; UNOP5332; UNOP5336); ponto 14 ( $23^{\circ} 17^{\prime} 40.0^{\prime}$ 'S , 4700'26.5'W). BRASIL. SÃo PAULO: Cabreúva, Serra do Japi, 14.V.2016, A.F. Tonetto s.n. (UNOP5343).

Características dos riachos $(\mathrm{n}=5)$ : turbidez 0,6-9,7 $\mu \mathrm{T}$ $(3,68)$; cor 9-60 $\mu \mathrm{H}(27,2) ; \mathrm{pH} 6,4-6,8(6,66)$; condutividade $16-41 \mu \mathrm{S}(25,2) ; \mathrm{N}$ Total $0,0-0,5 \mathrm{mg} / \mathrm{L}$ $(0,18)$; P Total $0,0-0,16 \mathrm{mg} / \mathrm{L}(0,06)$; fosfato $0,011-0,081 \mathrm{mg} / \mathrm{L} \mathrm{PO}_{4}(0,048)$; profundidade $10,2-29,4 \mathrm{~cm}(21,09)$, largura 200,4-705,2 cm $(393,4)$; velocidade da correnteza $0,51-0,71 \mathrm{~m} / \mathrm{s}(0,55)$ e ambientes parcialmente sombreados e sombreados.

Phormidium retzii Kützing ex Gomont, Ann. Sci. Nat. Bot., Sér. 7, 16: 175, 1892.

Figura 10

Filamentos emaranhados azul-esverdeados a azul escuro, com 7,7-9,3 $\mu \mathrm{m}$ diâm.; bainha fina e hialina, em geral ausente; tricomas não ramificados, em geral não constritos no septos; células com septos evidentes, conteúdo azul opaco com grânulos escuros pequenos, 4,9-11,7 $\mu \mathrm{m}$ compr. 4,8-9,3 $\mu \mathrm{m}$ diâm., compr./diâm. 0,7-2,4; célula apical truncada ou arredondada, 4,6-8,9 Hm compr., 4,9-7,3 diâm., compr./diâm. $0,7-1,4$.

Ocorrência: pontos 6 (2317'39.0”'S, 46 $\left.55^{\circ} 40.5^{\prime \prime} \mathrm{W}\right)$, 11 (2313'35.8”S, 4658'07.1”W). BRASIL. SÃo PaUlo: Jundiaí, Serra do Japi, 7-V-2016, A.F. Tonetto s.n. (UNOP5331; UNOP5336).

Características dos riachos $(\mathrm{n}=2)$ : turbidez 0,6-9,7 $\mu \mathrm{T}$ $(5,15)$; cor 9-60 $\mu \mathrm{H}(34,5) ; \mathrm{pH}$ 6,4-6,7 $(6,55)$; condutividade $17-41 \mu \mathrm{S}(29,0) ; \mathrm{N}$ Total $0,0-0,5 \mathrm{mg} / \mathrm{L}$ $(0,25)$; P Total $0,0-0,16 \mathrm{mg} / \mathrm{L}(0,08)$; fosfato $0,031-0,052 \mathrm{mg} / \mathrm{L} \mathrm{PO}_{4}(0,042)$; profundidade $10,2-15,4 \mathrm{~cm}(12,8)$, largura 200,4-524,0 cm $(362,2)$; velocidade da correnteza $0,51-0,53 \mathrm{~m} / \mathrm{s}(0,52)$ e ambientes parcialmente sombreados.

\section{Phormidium sp.}

Figura 11

Filamentos emaranhados azul-esverdeados, com 1,9-2,6 $\mu \mathrm{m}$ diâm.; bainha fina e hialina, às vezes ausente; tricomas não ramificados, constritos somente em algumas porções terminais; células com septos não evidentes, conteúdo azul-brilhante com alguns grânulos grandes, 1,4-2,3 $\mu \mathrm{m}$ compr., 1,4-2,5 $\mu \mathrm{m}$ diâm., compr./diâm. 1,0-2,0; célula apical arredondada, 1,5-2,1 $\mu \mathrm{m}$ compr., 1,7-2,9 diâm., compr./diâm.1,1-1,7.

Comentários taxonômicos: o material apresenta características morfológicas típicas do gênero Phormidium e remete ao grupo das espécies com ápices truncados e sem caliptra ("Grupo V" de Komárek \& Anagnostidis 2005). No entanto, as dimensões celulares dos espécimes encontrados são muito pequenas e não correspondem a nenhuma espécie já registrada. Estudos ultraestruturais e moleculares poderão definir o táxon e, potencialmente, verificar se trata-se de uma nova espécie.

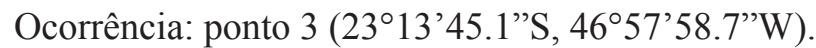
BRASIL. São Paulo: Jundiaí, Serra do Japi, 9-IV-2016, A.F. Tonetto s.n. (UNOP5322); Ponto 14. BRASIL. São Paulo: Cabreúva, Serra do Japi, 14-V-2016, A.F. Tonetto s.n. (UNOP5345).

Características dos riachos $(\mathrm{n}=2)$ : turbidez 0,7-2,0 $\mu \mathrm{T}$ $(1,16)$; cor 9-22 $\mu \mathrm{H}(16,6) ; \mathrm{pH} 6,4-6,8(6,63)$; Condutividade $15-21 \mu \mathrm{S}(17,3)$; N Total 0,0 mg/L; P Total $0,04-0,1 \mathrm{mg} / \mathrm{L}(0,06)$; fosfato $0,011-0,081 \mathrm{mg} / \mathrm{L}$ $\mathrm{PO}_{4}(0,036)$; profundidade $7,6-29,4 \mathrm{~cm}(19,8)$; largura 103,8-705,2 cm $(372,0)$; velocidade da correnteza $0,51-0,77 \mathrm{~m} / \mathrm{s}(0,61)$ e ambientes parcialmente sombreados e sombreados.

Scytonemataceae, Scytonema

Scytonema arcangeli Bornet et Flahault, Ann. Sci. Nat. Bot., Ser. 7, 5: 92, 1886.

Figura 12

Filamentos emaranhados formando tufos azulesverdeados a verde-brilhantes, com 12,0-14,1 $\mu \mathrm{m}$ diâm.; ramificações falsas, raras, simples e duplas; bainha evidente, hialina e espessa, às vezes com lamelação paralela e margens onduladas; tricomas não constritos ou levemente constritos; células com septos evidentes, conteúdo azul opaco a azulesverdeado; 5,5-10,1 $\mu \mathrm{m}$ compr., 8,3-10,7 $\mu \mathrm{m}$ diâm., 0,5-0,9; heterocitos raros, quadrados ou cilíndricos,

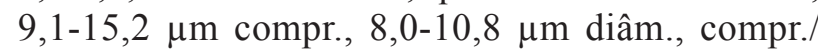
diâm.0,8-1,5.

Ocorrência: ponto 5 (2314'06.3”S, 4657'47.0”'W). BRASIL. São Paulo: Jundiaí, Serra do Japi, 9-IV-2016, A.F. Tonetto s.n. (UNOP5327). 
Características do riacho $(\mathrm{n}=1)$ : turbidez $0,9 \mu \mathrm{T}$; cor $11,0 \mu \mathrm{H}$; pH 6,0; condutividade $14,0 \mu \mathrm{S}$; N Total $0,0 \mathrm{mg} / \mathrm{L}$; P Total 0,02 mg/L; Fosfato 0,084 mg/L PO ; profundidade $22,0 \mathrm{~cm}$, largura $366,0 \mathrm{~cm}$; velocidade da correnteza $0,44 \mathrm{~m} / \mathrm{s}$ e ambiente sombreado.

Stigonemataceae, Stigonema

Stigonema mamillosum C. Agardh ex Bornet et Flahault, Ann. Sci. Nat. Bot. 5: 51-129. Figura 13

Filamentos emaranhados formando tufos prostrados azul-escuros a negros, com 33,2-56,6 $\mu \mathrm{m}$ diâm.; ramificações verdadeiras abundantes em série lateral de onde saem hormogônios; bainha firme, castanha ou hialina, homogênea, tricomas multisseriados, constritos, ápices atenuados e unisseriados; células com septos evidentes, conteúdo azul claro, grânulos pequenos, 7,4-16,8 $\mu \mathrm{m}$ compr., 7,4-13,0 $\mu \mathrm{m}$ diâm., compr./diâm. 0,6-1,4; heterocitos não observados.

Ocorrência: ponto 11 (2313'35.8”'S, 46 58'07.1”W). BRASIL. São Paulo: Jundiaí, Serra do Japi, 7-V-2016, A.F. Tonetto s.n. (UNOP5337).

Características do riacho $(\mathrm{n}=1)$ : turbidez $0,6 \mu \mathrm{T}$; cor 9,0 $\mu \mathrm{H}$; pH 6,4; condutividade $17,0 \mu \mathrm{S}$; N Total $0,0 \mathrm{mg} / \mathrm{L}$; P Total $0,0 \mathrm{mg} / \mathrm{L}$; fosfato $0,032 \mathrm{mg} / \mathrm{L} \mathrm{PO}_{4}$; profundidade $10,2 \mathrm{~cm}$, largura $524,0 \mathrm{~cm}$; velocidade da correnteza $0,53 \mathrm{~m} / \mathrm{s}$ e ambiente parcialmente sombreado.

Ochrophyta, Coscinodiscophyceae, Hydroseraceae, Hydrosera

Hydrosera whampoensis (A.F.Schwarz) Deby, J. Microgr. 15:209. 1891. ETriceratium whampoense A.F.Schwarz

Figuras 14, 15

Colônias filamentosas formando uma massa marrom no leito e aderidos às plantas aquáticas; células com valvas duplo-triangulares com ângulos arredondados e campos de poros apicais distintos; superfície valvar composta por aréolas irregulares ornamentais; células na vista pleural com 66,6-102,4 $\mu \mathrm{m}$ compr., 64,2-69,4 $\mu \mathrm{m}$ diâm., compr./ diâm. 0,9-1,5.

Ocorrência: ponto 7 (2317'22.4”S, 4656'25.9”W). BRASIL. São Paulo: Jundiaí, Serra do Japi, 7-V-2016, A.F. Tonetto s.n. (UNOP5333).
Características do riacho $(\mathrm{n}=1)$ : turbidez $5,3 \mu \mathrm{T}$; cor $39,0 \mu \mathrm{H}$; pH 7,0; condutividade $31,0 \mu \mathrm{S}$; N Total 0,4 mg/L; P Total 0,06 mg/L; fosfato 0,066 mg/L PO ; profundidade $27,8 \mathrm{~cm}$, largura $230,4 \mathrm{~cm}$; velocidade da correnteza $0,71 \mathrm{~m} / \mathrm{s}$ e ambiente sombreado.

Rhodophyta, Florideophyceae, Batrachospermaceae, Batrachospermum

Batrachospermum sp 1.

Figura 16, 17

Filamentos multisseriados pouco mucilaginosos e irregularmente ramificados, marrons; verticilos obcônicos, contíguos, com 163,3-356,0 $\mu$ m diâm.; internós com 110,8-411,7 $\mu \mathrm{m}$ compr.; fascículos primários di ou tricotomicamente ramificados, com 5-7 células; célula proximais do fascículo com 15,4-18,6 $\mu \mathrm{m}$ de compr., 7,8-11,1 $\mu \mathrm{m}$ diâm., células distais com 8,2-13,0 $\mu \mathrm{m}$ de compr. e 2,5-9,0 $\mu \mathrm{m}$ de diâm. Carposporófitos e carpogônio não observados.

Comentários taxonômicos: a identificação em nível específico não foi possível pela ausência dos carposporófitos e carpogônios nas amostras analisadas. A partir das demais características vegetativas apresentadas não foi possível indicar uma espécie potencial e aqui foi mantida a identificação em nível genérico Batrachospermum sp. podendo inclusive ser um dos gêneros relacionados (e.g. Sirodotia, por exemplo).

Ocorrência: ponto 16 (2313'45.5”S, 4652'29.0”W). BRASIl. São Paulo: Jundiaí, Serra do Japi, 14-V-2016, A.F. Tonetto s.n. (UNOP5349).

Características do riacho $(\mathrm{n}=1)$ : turbidez $15 \mu \mathrm{T}$; cor $72,0 \mu \mathrm{H}$; pH 7,1; condutividade 210,0 $\mu \mathrm{S}$; N Total $3,4 \mathrm{mg} / \mathrm{L}$; P Total $0,36 \mathrm{mg} / \mathrm{L}$; fosfato $0,33 \mathrm{mg} / \mathrm{L} \mathrm{PO}_{4}$; profundidade $21,0 \mathrm{~cm}$, largura $437,5 \mathrm{~cm}$; velocidade da correnteza $0,59 \mathrm{~m} / \mathrm{s}$ e ambiente aberto.

\section{Batrachospermum sp. 2}

Figuras 20-22

Filamentos multisseriados densamente mucilaginosos e irregularmente ramificados, azulescuros a negros; verticilos obcônicos, contíguos, 587,5-1069,7 $\mu \mathrm{m}$ diâm.; internós com 352,0-1088,8 $\mu \mathrm{m}$ compr.; fascículos primários retos, dicotomicamente ramificados, com 8-13 células; células proximais do fascículo com 35,7-36,0 $\mu \mathrm{m}$ compr., 5,7-7,5 $\mu \mathrm{m}$ diâm., células distais com 16,4-22,9 $\mu \mathrm{m}$ de comp., 4,2-8,2 $\mu \mathrm{m}$ diâm.; ramos carpogoniais retos, carpogônios claviformes, 22,1-40,9 $\mu \mathrm{m}$ compr., 3,5-6,7 $\mu \mathrm{m}$ diâm., compr./diâm. 5,8-6,9, carposporófitos não observados. 

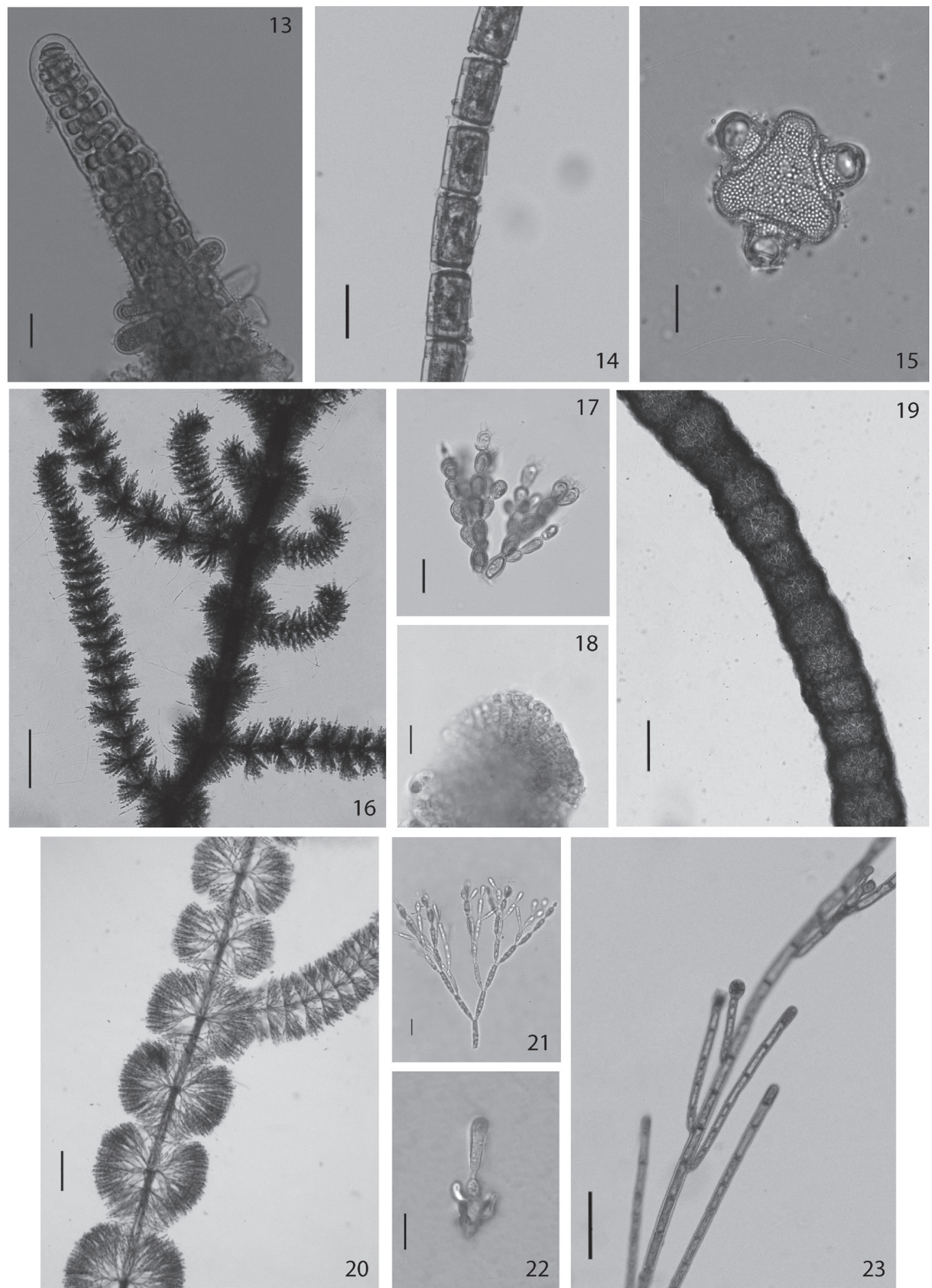

Figuras 13-23. Espécies de Cianobacteria, Rhodophyta e Ochrophyta da Serra do Japi. 13. Stigonema mamillosum. 14-15. Hydrosera whampoensis. 14. Filamento. 15. Vista valvar. 16-17. Batrachospermum cf. atrum. 16. Vista geral do talo com ramificações. 17. Ramo primário. 18. Hildenbrandia angolensis. 19. Filamento multisseriado de Compsopogon caeruleus. 20-22. Batrachospermum sp. 20. Vista geral do talo com ramificações. 21. Ramo primário. 22. Carpogônio mostrando tricogino. 23. Estagio esporofítico de Batrachospermum 'Chantransia'. Barra de escala $=20 \mu \mathrm{m}$.

Figures 13-23. Figures 13-23. Species of Cyanobacteria, Rhodophyta and Ochrophyta from Serra do Japi. 13. Stigonema mamillosum. 14-15. Hydrosera whampoensis. 14. Filament. 15. Valvar plane. 16-17. Batrachospermum cf. atrum. 16. General aspect of branching. 17. Primary branch. 18. Hildenbrandia angolensis. 19. Multisseriate filament of Compsopogon caeruleus. 20-22. Batrachospermum sp. 20. General aspect of branching. 21. Primary branch. 22. Carpogonium with tricogine. 23. Sporophytic stage of Batrachospermum 'Chantransia'. Scale bar $=20 \mu \mathrm{m}$. 
Comentários taxonômicos: a identificação em nível específico não foi possível pela ausência dos carposporófitos nas amostras analisadas. A partir das demais características vegetativas apresentadas não foi possível indicar uma espécie potencial e aqui foi mantida a identificação em nível genérico.

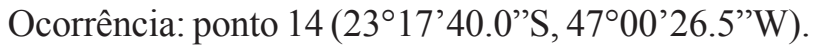
BRASIL. São Paulo: Cabreúva, Serra do Japi, 14-V-2016, A.F. Tonetto s.n. (UNOP5346)

Características do riacho $(\mathrm{n}=1)$ : turbidez $0,8 \mu \mathrm{T}$; cor 19,0 $\mu \mathrm{H}$; pH 6,8; condutividade $21,0 \mu \mathrm{S}$; N Total $0,0 \mathrm{mg} / \mathrm{L}$; P Total $0,04 \mathrm{mg} / \mathrm{L}$; fosfato $0,011 \mathrm{mg} / \mathrm{L} \mathrm{PO}_{4}$; profundidade $29,4 \mathrm{~cm}$, largura $307,2 \mathrm{~cm}$; velocidade da correnteza $0,51 \mathrm{~m} / \mathrm{s}$ e ambiente parcialmente sombreado.

\section{Estágio 'Chantransia'}

Figura 23

Filamentos unisseriados, ramificados, formando tufos arroxeados-escuros a negros; filamento composto por células cilíndricas, 33,1-109,0 $\mu \mathrm{m}$ compr., 13,2-29,8 $\mu \mathrm{m}$ diâm., compr./diâm. 2,3-4,6; ramificações se desenvolvem em ângulos $<25^{\circ} \mathrm{com}$ o eixo principal; monosporângios terminais, esféricos ou obovóides, 23,2-46,3 $\mu \mathrm{m}$ compr., 15,7-36,5 $\mu \mathrm{m}$ diâm., compr./diâm. 1-1,5.

Comentários taxonômicos: o estágio 'Chantransia' corresponde à fase esporofítica no ciclo de vida das Batrachospermales e pode estar associado a várias espécies.

Ocorrência: ponto $3\left(23^{\circ} 13^{\prime} 45.1^{\prime \prime} \mathrm{S}, 46^{\circ} 57^{\prime} 58.7^{\prime \prime} \mathrm{W}\right)$, ponto $4\left(23^{\circ} 14^{\prime} 07.8^{\prime \prime} \mathrm{S}, 46^{\circ} 57^{\prime} 51.2^{\prime \prime} \mathrm{W}\right)$, ponto 5 (2314'06.3”'S 4657'47.0”W). BRASIL. SÃO Paulo: Jundiaí, Serra do Japi, 9-IV-2016, A.F. Tonetto s.n. (UNOP5325; UNOP5326; UNOP5328); ponto $6\left(23^{\circ} 17^{\prime} 39.0^{\prime \prime} \mathrm{S}, 46^{\circ} 55^{\prime} 40.5^{\prime \prime} \mathrm{W}\right)$, ponto 9 (2317'03.9"S 46 56'20.6” W), ponto 11

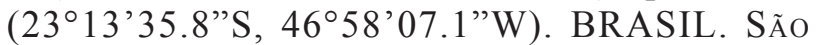
Paulo: Jundiaí, Serra do Japi, 7-V-2016, A.F. Tonetto s.n. (UNOP5330; UNOP5334; UNOP5338); ponto $14\left(23^{\circ} 17^{\prime} 40.0^{\prime}\right.$ 'S, $\left.47^{\circ} 00^{\prime} 26.5^{\prime \prime} \mathrm{W}\right)$, ponto 15 (2316'55.2'S, 4701'43.9'W). BRASIL. SÃo PAULO: Cabreúva, Serra do Japi, 14-V-2016, A.F. Tonetto s.n. (UNOP5342; UNOP5347).

Características dos riachos $(\mathrm{n}=8)$ : turbidez 0,6-9,7 $\mu \mathrm{T}(2,4)$; cor 9-60 $\mu \mathrm{H}(22,0)$; $\mathrm{pH} 6,0-7,3$ (6,5); condutividade 14-44 $\mu \mathrm{S}$ (24); N Total 0,0 $0,5 \mathrm{mg} / \mathrm{L}(0,15) ;$ P Total $0,0-0,19 \mathrm{mg} / \mathrm{L}(0,07)$; fosfato $0,009-0,113 \mathrm{mg} / \mathrm{L} \mathrm{PO}_{4}(0,057)$; profundidade
10,2-29,4 cm (21,1); largura 200,4-705,2 cm (368,2); velocidade da correnteza $0,44-0,93 \mathrm{~m} / \mathrm{s}(0,60)$ e ambientes parcialmente sombreados e sombreados.

Compsopogonaceae, Compsopogonaceae, Compsopogon

Compsopogon caeruleus (Balbis ex C.Agardh) Montagne, Algues Expl. Sc. Algerie, Bot. 1: 154. 1846. $\equiv$ Conferva caerulea Balbis ex C.Agardh. Figura 19

Filamentos multisseriados robustos, mucilaginosos, azul-escuros a negros; talo heterotríqueo, sistema prostrado firmemente aderido ao substrato, em forma de disco, filamentos eretos ascendem do sistema prostrado; filamento principal formado por células axiais doliformes, com

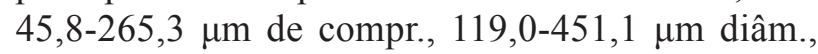
compr./diâm. 0,3-0,7, rodeadas por camadas de células corticais poligonais com 17,7-39,6 $\mu \mathrm{m}$ de diâm.; ápices unisseriados; monosporângios não observados.

Ocorrência: ponto 16 (231' 45.5'S, 4652'29.0”'W). BRASIL. São Paulo: Jundiaí, Serra do Japi, 14-V-2016, A.F. Tonetto s.n. (UNOP5348).

Características do riacho $(\mathrm{n}=1)$ : turbidez $15 \mu \mathrm{T}$; cor $72 \mu \mathrm{H}$; pH 7,1; condutividade 210,0 $\mu \mathrm{S}$; N Total 3,4 $\mathrm{mg} / \mathrm{L}$; P Total $0,36 \mathrm{mg} / \mathrm{L}$; fosfato $0,33 \mathrm{mg} / \mathrm{L} \mathrm{PO}_{4}$; profundidade $21,0 \mathrm{~cm}$, largura $437,5 \mathrm{~cm}$; velocidade da correnteza $0,59 \mathrm{~m} / \mathrm{s}$ e ambiente aberto.

\section{Hildenbrandiaceae, Hildenbrandia}

Hildenbrandia angolensis Welwitsch ex West \& G.S.West, J. Bot. 35: 3, 1897.

Figura 18

Filamentos prostrados, firmemente aderidas às rochas, formando manchas circulares ou irregulares de coloração enegrecida, as vezes avermelhada; filamentos eretos densamente agregados, curtos e pouco ramificados; células com 4,8-8,5 $\mu \mathrm{m}$ diâm.; gemas não observadas.

Ocorrência: ponto 3 (2313'45.1'S, 46 57'58.7'W), ponto 5 ( $\left.23^{\circ} 14^{\prime} 06.3^{\prime} \mathrm{S}, 46^{\circ} 57^{\prime} 47.0^{\prime \prime} \mathrm{W}\right)$. BRASIL. SÃo Paulo: Jundiaí, Serra do Japi, 9-IV-2016, A.F. Tonetto s.n. (UNOP5320; UNOP5323).

Características dos riachos $(\mathrm{n}=3)$ : turbidez 0,9-2,0 $\mu \mathrm{T}(1,3)$; cor 9-20 $\mu \mathrm{H}(13,3) ; \mathrm{pH} 6,0-6,8$ $(6,4)$; condutividade $14-20 \mu \mathrm{S}(16,6) ; \mathrm{N}$ Total 0,0-0,6 mg/L (0,2); P Total 0,02-0,05 mg/L (0,03); 
fosfato $0,081-0,084 \mathrm{mg} / \mathrm{L} \mathrm{PO}_{4}(0,08)$; profundidade $8,2-22,6 \mathrm{~cm}(17,6)$, largura 244-705,2 cm $(438,5)$; velocidade da correnteza $0,30-0,53 \mathrm{~m} / \mathrm{s}(0,45)$ e ambientes sombreados.

\section{Discussão}

O número total de táxons encontrados no presente estudo (16 no total, riqueza relativa de 1,0 táxon por ponto de amostragem) corresponde ao de trabalhos realizados em outras regiões usando metodologia semelhante: 1,4 táxons por ponto em Peres et al. (2008), 1,5 em Krupek et al. (2008), 1,1 em Branco et al. (2009), 0,9 em Necchi et al. (1995). Também corresponde ao encontrado em região de Mata Atlântica no Estado de São Paulo (mesmo bioma da área aqui amostrada) com 0,8 táxons por ponto de amostragem (Branco \& Necchi 1996). Essas regiões citadas possuem riachos predominantemente sombreados, condição similar a observada nos ambientes da Serra do Japi. Por outro lado, quando o total de espécies é comparado com levantamentos florísticos de regiões brasileiras com riachos mais iluminados, o número de táxons encontrado no presente estudo pode ser considerado baixo. Por exemplo, Necchi et al. (2008) em uma região que envolve campos de altitude encontraram uma riqueza relativa de 2,1 táxons por ponto de amostragem, enquanto Branco et al. (comunicação pessoal) encontraram 3,4 táxons por ponto em áreas de campos abertos no sul do Brasil. Assim, biomas com riachos de dossel aberto tendem a sustentar uma maior riqueza de espécies de macroalgas (como demostrado para algas verdes em Peres et al. 2017).

Nesse mesmo contexto, a condição sombreada da Serra do Japi pode explicar também o maior número de espécies de cianobactérias registrado na região. É recorrente o padrão de um número elevado de cianobactérias em relação aos outros grupos nos biomas florestais (e.g. Branco \& Necchi 1996, 1998, Peres et al. 2008, Krupek et al. 2008). Os representantes desse grupo algal apresentam um tipo de adaptação cromática que permite a presença, muitas vezes predominante, de espécies de cianobactérias independentemente do nível de luminosidade (Necchi 2004, Tonetto et al. 2012). Além disso, Branco et al. (2017) demonstraram que representantes desse grupo de algas apresentaram altos valores da razão fotossíntese/respiração em uma ampla variedade de tipos de riachos, mesmo quando comparados com outros grupos algais, ou mesmo espécies de briófitas e macrófitas aquáticas. Assim, as cianobactérias podem apresentar vantagens ecológicas que superam algumas limitações ambientais, como, por exemplo, o sombreamento e, permite, portanto, uma importante contribuição a produtividade de riachos tropicais mesmo em diferentes tipos de paisagens (Branco et al. 2017).

Outro aspecto sobre a diversidade de macroalgas da serra do Japi é que nenhuma espécie nova ou registro novo para as regiões tropicais foi observado. Apesar das espécies de macroalgas apresentarem distribuição reconhecidamente restrita (Branco et al. 2009), com aparentemente forte influência de características locais particulares (similar ao observado no presente trabalho), reduzir o déficit Linneano (Bini et al. 2006) parece ser um grande desafio quando se estuda esse grupo de organismos. Talvez porque algas, mesmo as macroalgas, podem ser consideradas pequenas, e organismos com tamanho reduzido tendem a apresentar distribuição espacial muito ampla (Fenchel \& Finlay 2004). Sendo assim, o endemismo pode ser bastante raro para espécies de algas de riachos. Neste contexto, a contribuição do presente estudo quanto à redução do déficit Wallaceano (Bini et al. 2006) de macroalgas lóticas torna-se especialmente importante para compreender as variações das espécies em relação aos eventuais impactos antrópicos sobre a diversidade desses organismos. Assim, aumentar o conhecimento sobre a distribuição espacial das macroalgas de riachos pode fornecer informações valiosas para construção de estratégias de conservação de recursos hídricos.

De modo geral, o registro da ocorrência das espécies de macroalgas lóticas na Serra do Japi amplia o conhecimento sobre a distribuição geográfica e a diversidade desses organismos. Assim, o estudo contribui para uma maior compreensão sobre a biogeografia de macroalgas de riachos, podendo ainda servir como fonte de dados para futuras questões ecológicas e revisões taxonômicas sobre algas de riachos.

\section{Agradecimentos}

Os autores agradecem a Prefeitura de Jundiaí e a base ecológica da Serra do Japi pelo apoio proporcionado na realização desse estudo. Em especial ao experiente guia da reserva Carlos Magno Pezzatto, que direcionou a equipe para os pontos de coleta com grande conhecimento. Ainda, agradecemos a UNIP pelo apoio ao projeto de pesquisa desenvolvido (Processo: 7-02-986/2016). 


\section{Literatura citada}

APHA. 1998. Standard Methods for the Examination of Water and Wastewater. 20 th edition. American Public Health Association, Washington D.C.

Bini, L.M., Diniz-Filho, J.A.F., Rangel, T.F.L.V.B., Bastos, R.P. \& Pinto, M.P. 2006. Challenging Wallacean and Linnean shortfalls: knowledge gradientes and conservation plannig in a biodiversity hotspot. Diversity and Distributions. 12: 475-482.

Branco, C.C.Z. \& Necchi Júnior, O. 1996. Survey of stream macroalgae of eastern Atlantic Rainforest of São Paulo State, southeastern Brazil. Algological Studies 80: 35-57.

Branco, C.C.Z., Branco, L.H.Z., Moura, M.O. \& Bertusso, F.R. 2005. The sucession dynamics of a macroalgal community after a flood disturbance in a tropical stream from São Paulo State, Southeastern Brasil. Revista Brasileira de Botânica 28: 267-275.

Branco C.C.Z., Necchi Junior, O. \& Peres, C.K. 2010. Effects of artificial substratum types and exposure time on macro-algal colonization in a tropical stream. Fundamental and Applied Limnology/Archiv fur Hydrobiologie 178: 17-27.

Branco, C.C.Z., Krupek, R.A. \& Peres, C.K. 2009. Ecological distribution of stream macroalgal communities from mid-western region of Paraná State, Southern Brazil: evidence of the importance of local scale variation. Brazilian Archives of Biology and Technology. 52: 379-386.

Branco, C.C.Z., Peres, C.K., Krupek, R.A. \& Bertusso, F.R. 2009. Macroalgas de ambientes lóticos da região Centro-Oeste do Paraná, região Sul do Brasil. Biota Neotropica 9: 1-9.

Branco, C.C.Z., Riolfi, T.A., Crulhas, B.P., Tonetto, A.F., Bautista, A.I.N. \& Necchi Junior, O. 2017. Tropical lotic primary producers: Who has the most efficient photosynthesis in low-order stream ecosystems? Freshwater Biology 62: 1623-1636.

Branco, L.H.Z. \& Necchi Júnior, O. 1998. Distribution of macroalgae in three tropical drainage basins of southeastern Brazil. Archiv für Hydrobiologie 142: 241-256.

Branco, L.H.Z., Necchi Júnior, O. \& Branco, C.C.Z. 1999. Cyanophyta from lotic ecosystems of São Paulo State, southeastern Brazil. Algological Studies 94: 63-87.

Brito, E.F., Moulton, T.P., Souza, M.L. \& Bunn, S.E. 2006. Stable isotope analysis indicates microalgae as the predominant food source of fauna in a coastal Forest stream, south-east Brazil. Austral Ecology 31: 623-633.

Brown, J.H. \& Lomolino, M.V. 1998. Biogeografia. Sinauer Associates, USA.
Cardoso-Leite, E. 2000. A vegetação de uma Reserva Biológica Municipal: contribuição ao manejo e à conservação da Serra do Japi, Jundiaí, SP. Tese de Doutorado, Universidade Estadual de Campinas, Campinas.

Cardoso-Leite, E., Pagani, M.I., Hamburger, D.S. \& Monteiro, R.R. 2002. Fitofisionomia, fitossociologia e conservação da vegetação na Reserva Biológica Municipal da Serra do Japi, Jundiaí, SP. Naturalia 27: $165-200$.

Dias, I.C.A. 1992. Algas continentais do Estado do Rio de Janeiro, Brasil: Oedogoniaceae e Zygnemaceae. Hoehnea 19: 51-63.

Dubois, A. 2003. The relationship between taxonomy and conservation biology in the century of extinctions. Comptes Rendus Biologies 326: S9-S21.

Fenchel, T. \& Finlay, B.J. 2004. The Ubiquity of Small Species: Patterns of Local and Global Diversity, BioScience 54: 777-784.

Guiry, M. \& Guiry, G. 2018. AlgaeBase. World-wide Electronic Publication. National University of Ireland, Galway. Disponível em http://www.algaebase.org. (acesso em 20-II-2018).

Hooper, D.U. et al. 2005. Effects of biodiversity on ecosystem functioning: a consensus of current knowledge. Ecological Monographs 75: 3-35.

Hu, B.F. \& Xie, S.L. 2006. Effect of seasonality on distribution of macroalgae in a stream system (Xin'an Spring) in Shanxi Province, North China. Journal of Integrative Plant Biology 48: 889-896.

Krupek, R.A., Branco, C.C.Z. \& Peres, C.K. 2008. Levantamento florístico das comunidades de macroalgas da bacia do Rio das Pedras, região centro-sul do Estado do Paraná, Sul do Brasil. Hoehnea 35: 189-208.

Loreau, M. Oteng-Yeboah, A., Arroyo, M.T.K., Babin, D., Barbault, R., Donoghue, M., Gadgil, M., Häuser, C., Heip, C., Larigauderie, A., Ma, K., Mace, G., Mooney, H. A., Perrings, C., Raven, P., Sarukhan, J., Schei, P., Scholes, R. J. \& Watson, R. T. 2006 Diversity without representation. Nature 442: 245-246.

Mace, G.M. 2004. The role of taxonomy in species conservation. Philosophical Transactions of the royal Society of London Series B-Biological Sciences 359: 711-719.

Muciolli, A.A.M. 1993. Caracterização e Diretrizes Gerais de Uso da Área de Proteção Ambiental (APA) da Serra do Japi, Jundiaí - SP. Trabalho de Conclusão de Curso, Universidade Estadual Paulista, Rio Claro.

Necchi Júnior, O., Branco, C.C.Z., Simões, R.C.G. \& Branco, L.H.Z. 1995. Distribution of stream macroalgae in northwest region of São Paulo State, southeastern Brazil. Hydrobiologia 299: 219-230. 
Necchi Júnior, O., Branco, C.C.Z. \& Branco, L.H.Z. 2000. Distribution of stream macroalgae in São Paulo State, southeastern Brazil. Algological Studies 97: 43-57.

Necchi Júnior, O., Branco, L.H.Z. \& Spezamiglio, D. 2008. Distribuição ecológica de comunidades de macroalgas de ambientes lóticos do Parque Nacional de Itatiaia (MG, RJ), Brasil. Brazilian Journal of Botany 31: 135-145.

Necchi Júnior, O. 2004. Light-related photosynthetic characteristics of lotic macroalgae. Hydrobiologia 525: 139-155.

Oliveira, R.C., Tonetto, A.T., Peres, C.K. \& Branco, C.C.Z. 2013. The influence of landscape on the spatial and temporal distribution of stream macroalgal communities of two types of subtropical biomes. Limnetica 32: 287-302.

Peres, C.K., Branco, C.C.Z. \& Krupek, R.A. 2008. Macroalgas de riachos da Serra da Prata, leste do Estado do Paraná, Sul do Brasil. Acta Botânica Brasilica 22: 333-344.

Peres, C.K., Tonetto, A.F., Garey, M.V. \& Branco, C.C.Z. 2017. Canopy cover as the key factor for occurrence and species richness of subtropical stream green algae (Chlorophyta). Aquatic Botany 137: 24-29.

Pinto, H.S. 1992. Clima da Serra do Japi in História Natural da Serra do Japi. Editora da Unicamp, Campinas, pp. 30-39.

Saka, M.N. 2009. Solanaceae da reserva biológica municipal da serra do Japi, Jundiaí, SP. Trabalho de Conclusão de Curso, Universidade Estadual Paulista, Rio Claro.
Sherwood, A.R. \& Sheath, R.G. 1999. Seasonality of macroalgae and epilithic diatoms in spring-fed streams in Texas, U.S.A. Hydrobiologia 390: 73-82.

Sheath, R.G. \& Cole, K.M. 1992. Biogeography of stream macroalgae in North America. Journal of Phycology 28: 448-460.

Sheath, R.G., Hamilton, P.B., Hambrook, J.A. \& Cole, K.M. 1989. Stream macroalgae of eastern boreal forest region of North America. Canadian Journal of Botany 67: 3553-3562.

Sodhi, N.S. \& Ehrlich, P.R. 2010. Conservation Biology for all. Oxford University Press, UK.

Strahler, A.N. 1957. Quantitative analysis of watershed geomorphology. Transactions of the American Geophysical Union, Washington. 38: 913-920.

Tonetto A.F., Branco, C.C.Z. \& Peres, C.K. 2012. The effects of irradiance and spectral composition on the establishment of macroalgae in streams. Annales de Limnologie-International Journal of Limnology 48: 363-370.

Tonetto, A.F., Cardoso-Leite, R., Peres, C. K., Bispo, P.C. \& Branco, C.C.Z. 2014. The effects of habitat complexity and hydraulic conditions on the establishment of benthic stream macroalgae. Freshwater Biology 59: 1687-1694.

Tonetto, A.F., Cardoso-Leite, R., Novaes, M.C \& Guillermo-Ferreira, R. 2015. The relationship between macroalgal morphological complexity and hydraulic conditions in stream habitats, Hydrobiologia 747: 33-41.

Wilson, E.O. 1992. The diversity of life. Belknap Press. Cambridge, UK. 